Proceedings

\title{
Do our patients really need telerehabilitation? Digital physical therapy of boys with Duchenne Muscular Dystrophy in the COVID-19 pandemic Gdansk, 30.12.2020
}

\author{
Agnieszka Sobierajska-Rek ${ }^{1 *}$, Łukasz Mański ${ }^{2}$, Joanna Jabłońska-Brudło ${ }^{1}$ and Karolina Śledzińska ${ }^{3}$ \\ 1 Department of Rehabilitation Medicine, Faculty of Health Sciences with Institute of Maritime and Tropical \\ Medicine, Medical University of Gdansk, Gdansk, Poland; address: Al. Zwyciestwa 30; 80-219 Gdansk, \\ Poland, e-mail: agnieszka.sobierajska-rek@gumed.edu.pl (A.S.-R.); jibrudlo@gumed.edu.pl (J.J.-B.) \\ 2 Department of Physical Therapy, Faculty of Health Sciences with Institute of Maritime and Tropical \\ Medicine, Medical University of Gdansk, Gdansk, Poland;address: ul.Debinki 7, 80-211 Gdansk, Poland, \\ e-mail: lmanski@gumed.edu.pl \\ 3 Department of Internal and Pediatric Nursing, Faculty of Health Sciences with Institute of Maritime and \\ Tropical Medicine, Medical University of Gdansk, Gdansk, Poland; address: ul.Debinki 7, 80-211 Gdansk, \\ Poland, e-mail: karolina.sledzinska@gumed.edu.pl \\ * Correspondence: agnieszka.sobierajska-rek@gumed.edu.pl
}

Publisher's Note: MDPI stays neutral with regard to jurisdictional claims in published maps and institutional affiliations.

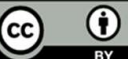

Copyright: $\odot 2020$ by the authors. Submitted for possible open access publication under the terms and conditions of the Creative Commons Attribution (CC BY) license (http://creativecommons.org/licenses /by/4.0/).

\begin{abstract}
The COVID-19 pandemic forced reorganization of the multidisciplinary healthcare system for Duchenne muscular dystrophy. Digital solutions seem to be optimal way for providing rehabilitation for this time. The aim of our study was to investigate whether it is possible to conduct respiratory physical therapy with the use of telerehabilitation methods in boys with Duchenne muscular dystrophy. Methods: The study was conducted during on-line conference 'DMD - let's be together'for Polish families with DMD. During the physical therapy panel we showed the video with the instructions of respiratory exercises: glossopharyngeal breathing, positive inspiratory pressure and positive expiratory pressure. All participants $(n=152)$ were asked to fill in the on-line survey evaluating the quality of the instructions, its acceptance and understanding. Results: The survey was filled in by $31(20.4 \%)$ participants, mean age of patients 13.8, $19(61.3 \%)$ were ambulant. The video was displayed 127 times. The overall mean rating of the session was $4.77 / 5$, intelligibility 4.74/5, $14(45.2 \%)$ patients declared that they had performed the exercises by themselves or with caregiver's assistance, all caregivers declared that it is possible to perform proposed exercises few times a week or daily, only two respondents replied for invitation for individual on-line session. Conclusions: Findings from the study show that respiratory telerehabilitation may be implemented in DMD patients, however the interest of digital rehabilitation among caregivers of DMD boys in Poland is low. The reason of this situation requires further research.
\end{abstract}

Keywords: DMD; children; telerehabilitation

\section{Introduction}

Duchenne muscular dystrophy (DMD) is the most common and severe neuromuscular disease affecting approximately 1 in 3500 persons worldwide. This incurable X-linked recessive disorder is caused by the mutation in the dystrophin gene leading to impaired production or function of dystrophin protein, which in turn, leads to progressive muscle weakness. Boys with DMD boys in the second decade of life lose ambulation, then the ability to stand or change position. The milestones in the disease progression are: lack of ambulation, loss of ability to use upper limbs, gastrointestinal problems resulting in malnutrition, heart failure, and respiratory insufficiency [1,2].

Historically, the cardiorespiratory system failure caused death before 18 years of 
age. Nowadays there is still no cure for DMD, but the appropriate treatment and medical multidisciplinary care can improve the quality of life of those patients and extend their life expectancy to more than 30 years of age [2].

Assessment and treatment of respiratory system is one of the most important problems for DMD patients. Progression of the disease and muscle weakness lead to progressive scoliosis. Massive deformity raises the risk of cardiopulmonary dysfunction [2]. The first signs of respiratory failure are obstructive sleep apnea (OSA) which causes chronic headaches, daytime drowsiness, episodes of distraction, difficulty of falling asleep and waking up. Adult DMD patients, especially with FVC $<60 \%$ with weak cough and airway clearance are at high risk of severe respiratory infections [3, 4].

The pandemic has influenced health care system in the entire world. Due to the fact that DMD patients are at risk of severe course of COVID-19 [5] the multidisciplinary healthcare team is seeking the safest way to provide constant care and treatment on the highest possible level. The innovative digital form of physical therapy which is easily available, free of charge and adjustable can support the daily routine of home training in the time of social distancing [6].

The purpose of the study was to investigate whether it is possible to conduct respiratory physical therapy with the use of telerehabilitation methods in boys with Duchenne muscular dystrophy.

\section{Materials and Methods}

The study included 152 boys with DMD (ambulatory and non-ambulatory), and it was conducted during the on-line conference "DMD-lets be together" for Polish families with DMD. During the physical therapy panel, we showed the video with the instruction of respiratory exercises: positive inspiratory pressure, glossopharyngeal breathing and positive expiratory pressure. The exercises could be performed at home without physiotherapists' assistance. Patients were encouraged to train every day, three times a day. On the video patients received following instructions:

1. Breath stacking (positive inspiratory pressure): patients were asked to take maximal inhale, then without exhaling, to take another 1-3 inhales, and hold for 5 seconds. Boys were instructed to repeat the exercise 5 times. The aim of the exercise was to increase lung volume. The same exercise was presented with the use of balloon - inflated balloon - patients were instructed to inhale the air from balloon, without exhaling and try to hold as much air in the lungs as possible - hold for 5 seconds and repeat for 5 times.

2. Glossopharyngeal breathing - patients were instructed to push series of small volumes of air by tongue and pharynx into the lungs by saying 'cat' in Polish - patients were encouraged to repeat the exercise for 5 times.

3. Positive expiratory pressure - inflating the balloon - patients were instructed to inflate the balloon by inhaling by nose and exhaling by mouth - the exercise should be repeated for 3 times. Caregivers were encouraged to try different types of balloons to find optimal elasticity of material (optimal resistance).

The video is still available in the internet https://www.youtube.com/watch?v=AEaxOsuJimU\&feature=youtu.be.

After the conference all participants $(n=152)$ were asked to fill in the on-line survey evaluating this home-based programme. The survey consisted of question about age, ambulation and upper limb functional status. Respondents were also asked to assess in six points scale general satisfaction, appropriateness of the exercises and intelligibility, were 0 meant the worse and 5 meant the best score. Moreover, in the questionnaire we asked whether the exercises were performed. The respondents assessed difficulty of the exercises and possibility to implement this treatment to daily routine. Additionally, caregivers were invited to leave an e-mail contact in case they needed expanded individual consultation or training. 
The study was conducted as a part of the Multidisciplinary Care Program for Patients with Duchenne Muscular Dystrophy performed by Rare Disease Centre (RDC), University Clinical Centre, Medical University of Gdańsk, Poland. The University Clinical Centre is a member of the TREAT NMD Alliance Neuromuscular Network.

The study was accepted by the Ethics Committee and assigned the number NKBBN/105/2018.

\section{Results}

The video with respiratory exercises was displayed 127 times in a first month. The on-line survey was filled by 31 (20,4\%) participants, mean age of patients 13.8 years old. $19(61.3 \%)$ were ambulant. Detailed ambulatory and upper limb functional status is presented in Table 1.

Table 1. Detailed ambulatory and upper limb functional status.

\begin{tabular}{|c|c|c|c|}
\hline Ambulatory status & $n=31$ & $\begin{array}{c}\text { Upper limb } \\
\text { functional status }\end{array}$ & $\mathrm{n}=31$ \\
\hline $\begin{array}{l}\text { Walks and climbs } \\
\text { stairs without assis- } \\
\text { tance }\end{array}$ & 9 & $\begin{array}{l}\text { Can abduct the arms } \\
\text { in a full circle until } \\
\text { they touch above the } \\
\text { head. }\end{array}$ & 16 \\
\hline $\begin{array}{l}\text { Walks and climbs } \\
\text { stairs with railing or } \\
\text { assistance }\end{array}$ & 10 & $\begin{array}{l}\text { Can raise arms above } \\
\text { head only by flexing } \\
\text { the elbow. }\end{array}$ & 10 \\
\hline \multirow[t]{2}{*}{ Uses a wheelchair } & \multirow[t]{2}{*}{12} & $\begin{array}{l}\text { Cannot raise hands to } \\
\text { the mouth but can use } \\
\text { hands to hold a pen or } \\
\text { pick up coins from the } \\
\text { table. }\end{array}$ & 2 \\
\hline & & $\begin{array}{l}\text { Cannot rise hands to } \\
\text { the mouth and has no } \\
\text { useful function of } \\
\text { hands. }\end{array}$ & 1 \\
\hline
\end{tabular}

The general rating of the on-line respiratory program was $4,77 / 5$, appropriateness 4,74/5, intelligibility 4,74/5.

The number of participants who tried to perform the exercise by themselves was 5 $(16,1 \%)$, with assistance 19 (61,2\%), 7 (22,6\%) did not perform the exercises (Figure 1.)

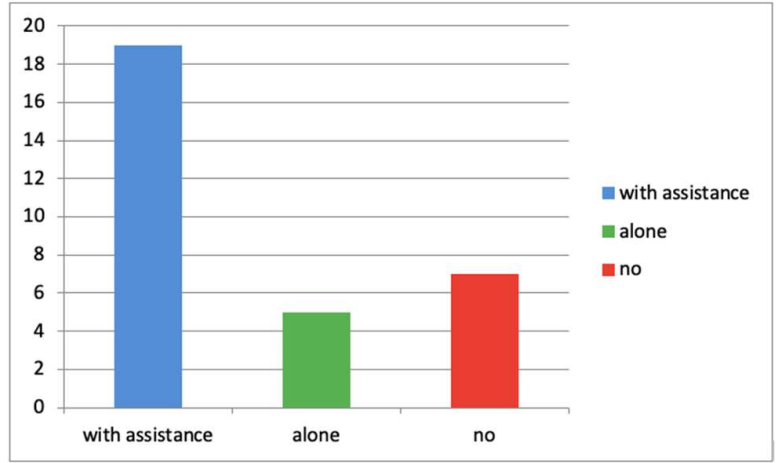

Figure 1. Number of participants who performed the exercises. 
Only one respondent declared that the patient was able to perform properly all exercises, 16 were able to perform all exercises after practicing, 14 declared that some exercises were too difficult for the patient.

Respondents declared that it is possible to implement respiratory exercise to home-based rehabilitation routine. Declared by caregivers/patients frequency of the exercises is presented in Figure 2.

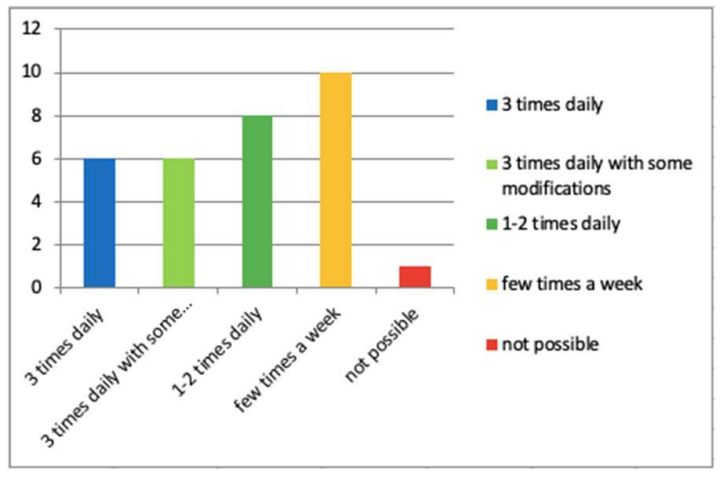

Figure 2. Declared frequency of the exercises.

Only two caregivers responded for the invitation for further individual consultation or assistance with exercise performance.

One respondent chose phone consultation - non-ambulatory 18-year-old patient the patient and his parents asked questions about symptoms of dyspnoea, eventual disturbing symptoms regarding respiratory condition demanding hospitalization, and the efficacy of home use of pulse oximeter. Parents were also worried because of cancelling regular multidisciplinary consultations caused by pandemic. However, they have admitted that the patients did not try to perform proposed respiratory exercises.

Another respondent chose e-mail consultation - the mother of 6-year-old ambulant boy reported that her son had difficulties with respiratory exercises and performing pulmonary function tests doing because of inability to perform forced expiration.

\section{Discussion}

Nowadays, multidisciplinary DMD healthcare team is facing the challenge to ensure consistency in care and treatment. Respiratory rehabilitation is one of the most pressing problems.

Before pandemic in 2015 the American Thoracic Society (ATS) and European Respiratory Society (ERS) suggested to perform telerehabilitation to increase availability of pulmonary rehabilitation to more patients [7].

Current studies concerning respiratory telerehabilitation report that this intervention is effective in reducing disability, improving quality of life and may reduce the risk of severe excerbations $[8,9,10]$. Moreover there is evidence that telerehabilitation in pediatric population may be as effective as face-to-face treatment [11]. However, to our knowledge this is the first study concerning respiratory digital in DMD.

Unfortunately, we noticed low response rate to the on-line survey conducted after conference, as well as low amount of patients who made an effort to perform proposed exercises.

Our previous study concerning digital physical therapy showed similar low response rate to the online physical therapy workshops. Moreover, the survey also showed that $57.8 \%$ of DMD patients included to abovementioned study felt overloaded with home schooling responsibilities [6]. This may be the reason of low response to the survey and/or treatment provided on-line. Another reason may be related to not enough trust to healthcare interventions conducted indirectly. It has to be mentioned that telerehabilita- 
tion is a new method in Poland and is not widely known among parents/caregivers of DMD patients.

Similarly, low resposne rate is described in telerehabiliation studies involving patients with different disorders. The study of Hensen concerning pulmonary telerehabilitation of 134 patients with COPD, revealed that only $12.19 \%$ of group that met inclusion criteria managed to complete intervention protocol [8].

Ratings of the quality and understanding of the instructions as well as acceptance of proposed exercises programme indicate that it is possible to implement such intervention to home-based rehabilitation daily routine. Additionally, our results revealed that most patients performed the exercises with the caregiver's assistance. This observation may be a hint for future design of on-line interventions. Promising findings show that majority of respondents declared that it would be possible to exercise more frequently that 1-2 times a day, however the question about effectiveness of the home-based respiratory exercises in this group of patients remains open.

In summary, online rehabilitation during COVID pandemic as well as conventional care of DMD boys should always rely on individual approach taking into consideration patients' age, functional abilities, cognitive limitations and level of cooperation.

\section{Conclusion}

The study shows that respiratory telerehabilitation may be implemented in DMD patients, however it should include caregiver's assistance. The interest of digital rehabilitation among caregivers of DMD boys in Poland is low which requires further research. Moreover, there is a need for well designed randomized clinical trial assessing effectiveness of proposed interventions.

Supplementary Materials: The video with respiratory exercises is available online at https://www.youtube.com/watch?v=AEaxOsuJimU\&feature=youtu.be, Video S1: Respiratory exercises.

Author Contributions: Conceptualization, A.S.-R., and Ł.M.; methodology, A.S.-R.; validation, A.S.-R. and J.J.-B.; formal analysis, A.S.-R., Ł.M.; investigation, A.S.-R. and Ł.M.; resources, K.Ś.; data curation, A.S.-R. and Ł.M.; writing-original draft preparation, A.S.-R., Ł.M., J.J.-B. and K.Ś. writing - review and editing, A.S.-R., Ł.M., J.J.-B. and K.Ś; visualization, A.S.-R., Ł.M.; supervision, J.J.-B.; project administration, A.S.-R. All authors have read and agreed to the published version of the manuscript.

Funding: This research received no external funding.

Institutional Review Board Statement: The study was conducted according to the guidelines of the Declaration of Helsinki and approved by the Ethical Committee of Independent Bioethics Committee for Scientific Research at Medical University of Gdańsk: NKBBN/105/2018.

Informed Consent Statement: Patient consent was waived due to the reason of anonymity of the survey.

Data Availability Statement: In this section, please provide details regarding where data supporting reported results can be found, including links to publicly archived datasets analyzed or generated during the study. Please refer to suggested Data Availability Statements in section "MDPI Research Data Policies" at https://www.mdpi.com/ethics. You might choose to exclude this statement if the study did not report any data.

Conflicts of Interest: The authors declare no conflict of interest.

\section{References}

1. Birnkrant D.J.; Bushby K.; Bann C.M. et al. Diagnosis and management of Duchenne muscular dystrophy, part 1: diagnosis, and neuromuscular, rehabilitation, endocrine, and gastrointestinal and nutritional management. Lancet Neurol. 2018, 17(3), 251-267.

2. Wasilewska E.; Małgorzewicz S.; Sobierajska-Rek A.; Jabłońska-Brudło J. Transition from Childhood to Adulthood in Patients with Duchenne Muscular Dystrophy. Medicina 2020, 56(9), 426. doi:10.3390/medicina56090426 
3. Birnkrant D.J.; Bushby K.; Bann C.M.; et al. Diagnosis and management of Duchenne muscular dystrophy, part 2: respiratory, cardiac, bone health, and orthopaedic management. Lancet Neurol. 2018, 17(4), 347-361. doi:10.1016/S1474-4422(18)30025-5

4. LoMauro A.; D'Angelo M.G.; Aliverti A. Assessment and management of respiratory function in patients with Duchenne muscular dystrophy: current and emerging options. Ther Clin Risk Manag. 2015, 11, 1475-1488. doi:10.2147/TCRM.S55889

5. Veerapandiyan A.; Wagner K.R.; Apkon S. et al. The care of patients with Duchenne, Becker, and other muscular dystrophies in the COVID-19 pandemic. Muscle and Nerve. 2020, 62, 41-45. doi:10.1002/mus.26902

6. Sobierajska-Rek A.; Mański Ł.; Jabłońska-Brudło J.; Śledzińska K.; Ucińska A.; Wierzba J. Establishing a telerehabilitation program for patients with Duchenne muscular dystrophy in the COVID-19 pandemic. Wien Klin Wochenschr. Published online 2020. doi:10.1007/s00508-020-01786-8

7. Rochester C.L.; Vogiatzis I.; Holland A.E. et al. An Official American Thoracic Society/European Respiratory Society Policy Statement: Enhancing Implementation, Use, and Delivery of Pulmonary Rehabilitation. Am J Respir Crit Care Med. 2015, 192(11), 1373-1386. doi:10.1164/rccm.201510-1966ST

8. Hansen H.; Bieler T.; Beyer N. et al. Supervised pulmonary tele-rehabilitation versus pulmonary rehabilitation in severe COPD: a randomised multicentre trial. Thorax. 2020, 75(5), 413-421. doi:10.1136/thoraxjnl-2019-214246

9. Tousignant M.; Marquis N.; Pagé C. et al. In-home Telerehabilitation for Older Persons with Chronic Obstructive Pulmonary Disease: A Pilot Study. Int J Telerehabilitation. 2012, 4(1), 15-24. doi:10.5195/ijt.2012.6083

10. Bourne S.; DeVos R.; North M. et al. Online versus face-to-face pulmonary rehabilitation for patients with chronic obstructive pulmonary disease: randomised controlled trial. BMJ Open. 2017, 7(7), e014580. doi:10.1136/bmjopen-2016-014580

11. Camden C.; Pratte G.; Fallon F.; Couture M.; Berbari J.; Tousignant M. Diversity of practices in telerehabilitation for children with disabilities and effective intervention characteristics: results from a systematic review. Disabil Rehabil. 2020, 42(24), 3424-3436. doi:10.1080/09638288.2019.1595750 